\title{
Los Centros de Reconocimiento de Conductores veinte años después
}

\section{The Assessment of Drivers Centres twenty years later}

F. TORTOSA*

L. MONTORO*

J. ROCA**

C. CIVERA*

\section{RESUMEN}

Si bien existe una tradición española en la aplicación de los conocimientos psicológicos a la prevención de accidentes de circulación, que arranca desde la década de 1920, no se convirtió en un fenómeno de masas hasta mediados de los años 80 cuando, al extenderse desde los conductores profesionales a todos los conductores, llegó a un número creciente de españolas y españoles. Esta acción preventiva, que fue el primer rol profesional de intervención psicológica legalmente regulado en España, definido incluso antes de la existencia formal de una auténtica práctica académica en Psicología, es hoy una destacada fuente de empleo para los titulados en psicología y goza de plena actualidad, como demuestra su reciente inclusión dentro del sistema de permiso por puntos. Los protagonistas de la historia fueron, primero, los psicotécnicos en Centros públicos evaluando conductores profesionales, y luego los psicólogos en Centros privados explorando a todos los aspirantes a obtener o renovar los permisos. La selección ha sido el hilo conector de lo que ha dado en llamarse el "Modelo Español" de Seguridad Vial. Se puede establecer varias etapas en el desarrollo del mencionado modelo, coincidentes en una gran parte con el desarrollo y consolidación de una Ciencia Psicológica española autónoma y con los cambios de paradigmas y tecnologías. El actual Reglamento General de Conductores mantiene el

\footnotetext{
* Cátedra de Prevención de Riesgos en los Comportamientos Viales. Mutua Madrileña-UVEG.

** Instituto de Tráfico y Seguridad Vial (INTRAS). Universitat de València (UVEG).
} 
mismo patrón preventivo legalizado en 1934. Tras casi 100 años de vida y 25 de acción sobre masas, cabía preguntarse por su validez y utilidad, y eso es precisamente, apoyándose en datos empiricos, lo que pretende contestar este articulo.

\title{
PALABRAS CLAVE
}

Selección de conductores, Perfil profesional, Psicotecnia, Representación social, Prevención accidentes tráfico.

\begin{abstract}
It is appropriate to talk about a Spanish tradition in the application of psychology to traffic accidents prevention, dating back to the 1920s. It was the first professional area of psychological intervention to be legally regulated in Spain, even before the formal existence of an epistemic practice in psychology. From the 1930s on, without any interruption, there has been a prevailing preventive model, in spite of the different political regimes that have governed in Spain. First the psychotechnicians in public centres assessing professional drivers, and later the psychologists in private centres with all drivers have been the protagonists in the story, the connecting thread being what has been called the Spanish Model of drivers assessment. We can establish various different steps in the development of the Spanish Model in Psychological Assessment of Drivers, in a big part coincident with the evolution of the Psychological Science and the Spanish psychology. The current "Reglamento General de Conductores", who regulate driving licence, required from all of candidates to obtain/renewal driving licence submitted to physical and psychological examination in private centres to obtain driving licence. Also, this examination is required to those drivers who have lost their licence as a consequence of the penalty point system and are trying to recover it. The "Reglamento General de Conductores" maintained psychological test and a preventive pattern, legalized at 1934. We offered an empirical analysis of its activity and the validity of the professional intervention in its different dimensions. The selection of drivers has played a key role in the building of Modern professional Spanish psychology, and is today the oldest living representative role in the field.
\end{abstract}

\section{KEY WORDS}

Drivers selection, Professional role, Psychotechnics, Social representation, Traffic accident prevention. 


\section{INTRODUCCIÓN}

El desarrollo del transporte (aéreo, naval, ferroviario), y sobre todo el uso masivo del automóvil, ha transformado las vidas de los seres humanos en el globalizado mundo en el que nos hallamos instalados. Ha producido transformaciones que afectan la vida humana, la ecologia, la economía, la cultura, el ocio, la sociedad, e incluso la propia definición de dependencia, generando un nuevo tipo de personas dependientes, las que necesitan de otros para moverse en unos entornos cada vez más pensados para que circulen vehículos, y menos para que deambulen personas. En definitiva, los vehículos han cambiado drásticamente la forma de vivir, $y$, lamentablemente, también la de morir.

La siniestralidad vial ha ido, con sus crecientes cifras y su incidencia diferencial en grupos de edad y género, definiendo un problema de salud pública de grandes magnitudes. En el famoso Informe Gerondeau, elaborado en 1991 por un grupo de expertos sobre la política europea en seguridad vial se escribía: “(...) desde la firma del Tratado de Roma, el tráfico ha matado a más de dos millones de habitantes en los países miembros de la Comunidad Europea y ha herido a más de 40 millones de ellos, lo que constituye un verdadero balance de guerra." También como en las guerras sus consecuencias son especialmente devastadoras entre los sectores más jóvenes de la población, donde sólo el SIDA es un serio competidor en términos de años potenciales de vida perdidos.

La no aceptación racional de la inevitabilidad de los siniestros viales y el hecho de que la mayor parte de ellos se produzcan por fallos humanos (Montoro, Toledo, Lijarcio y Roca, 2006), llevó, desde principios de siglo, a un maridaje entre la Psicologia/Psicotecnia y la Segu- ridad Vial, que asumieron y potenciaron prácticamente todos los países desarrollados del mundo, aunque ha conocido historias muy distintas en los diferentes países (Gundlach, 1998; Barjonet y Tortosa, 2001; Tortosa y Montoro, 2002; Tortosa, Barjonet, Civera y Montoro, 2003).

La respuesta generalizada para prevenir la accidentalidad vial, defendida abiertamente desde las primeras Conferencias Internacionales de Psicotecnia que se iniciaron allá por los años 20 del pasado siglo, fue el desarrollo de dictámenes de capacitación (exámenes de aptitud) exigibles a los conductores profesionales. Para efectuar aquellos primeros exámenes se recurrió básicamente a la psicología experimental sensorial, que utilizaba instrumentos de medida que evaluaban diversas funciones sensoriales y a través de aquellas el estado de funciones psíquicas superiores (enfoque diferencial-psicofísico), y a la medición de ciertos factores personales (enfoque diferencial-psicométrico). España, tomando como guía los modelos de examen alemán y francés (Serra y Tortosa, 2004), se incorporó al proceso de adecuar, o elaborar ex novo, pruebas de evaluación para emitir esos dictámenes.

De hecho, tras la I Guerra Mundial, se había definido ya un campo y rol de actuación profesional, la (entonces) Psicotecnia aplicada al tráfico y el transporte de personas y mercancías (tranvías, trenes, autobuses, camiones, coches, aviones, barcos), y al manejo de vehículos bélicos. Ese rol ha ido perfilándose hasta definir una (hoy) "Psicología del Tráfico y de la Seguridad (p.e., Vial, Aeronáutica, Ferroviaria, Naval)" (Hoyos, 1992), que, en nuestro caso, ha tenido como núcleo el llamado "modelo español" de Seguridad Vial (Capdevila y Saiz, 1998; Civera, Pastor y Alonso, 2003; Saiz y Saiz, 1998; Serra y Tortosa, 2004; 
Tortosa, Esteban, Tejero, y Molero, 1995). La situación es tal que hoy es el referente de uno de los ocho perfiles profesionales definidos por el Colegio Oficial de Psicólogos (1998), y uno de los que más empleo genera según la Encuesta de Actividad Profesional que, periódicamente realiza el propio Colegio (Santolaya, Berduyas y Fernández-Hermida, 2002).

El pilar básico del mencionado modelo de intervención profesional ha sido sin duda la labor preventiva que se realiza hoy, desde los Centros (privados) de Reconocimiento como ayer desde los Institutos (públicos) de Psicología y Psicotecnia. El objetivo de la exploración era, y es, realizar, individualmente, una valoración del estado psicofísico actual de cada aspirante, con arreglo a unos parámetros de normalidad (fisica y psíquica), obtenidos mediante la utilización de instrumentos y pruebas homologados. Con ello se pretende más que predecir el mayor o menor riesgo de un potencial conductor de sufrir o producir conflictos 0 accidentes, establecer (o prever) el nivel de deterioro, o de posible deterioro, de dimensiones psicofísicas relevantes para un adecuado desempeño como conductor, en el convencimiento de la posible influencia de dicho estado sobre la calidad de la conducción.

Millones de personas pasan cada años por esos Centros de Reconocimiento, millones son pues los españoles y españolas explorados por médicos, oftalmólogos y psicólogos "del tráfico", y qué pasa con esas exploraciones es la pregunta que muchos se hacen, o lo que es lo mismo, muchos se preguntan si sirven, si son útiles, algo que, muchas veces en sentido negativo, comentan algunos medios, y no siempre con datos en la mano. Nosotros pretendemos adelantar una respuesta a partir de la revisión de cientos de miles de datos analizados.

\section{UNOS POCOS DATOS SOBRE LA MAGNITUD DEL PROBLEMA}

En España las dimensiones del fenómeno circulatorio son colosales. Comencemos por ubicar las magnitudes del problema con unas pocas comparaciones que nos sitúen. En el último trimestre de 1900 se matriculaban 3 coches en España, mientras que en el último trimestre de 2005 se hacia lo propio con casi 553.000 coches, llegando a los 30.000 millones de vehículos (incluyendo ciclomotores), 681 por cada 1000 habitantes, más de 800 en el tramos de los 30 a los 40 años, ¡casi a vehículo por persona! Circulan por cerca de doscientos mil kilómetros de carreteras españolas, conducidos por más de veintitrés millones de conductores, la mitad con menos de 40 años.

El Observatorio Nacional de Seguridad Vial, para ese mismo año de 2005 (102 muertos por millón de habitantes), señala que ha habido 91.187 accidentes de circulación con victimas, con 137.251 victimas, de ellos 4.442 muertos dentro de los 30 días siguientes al accidente y 21.859 heridos graves, es decir heridos que necesitaron más de 24 horas de hospitalización. Aún siendo muy altas, estas cifras suponen una reducción de más del $5 \%$ respecto de las de 2004 (110 muertos por millón de habitantes), y del $18 \%$ respecto de las de 2003 (128 muertos por millón de habitantes): Más de un millar de personas fallecidas menos; y ello en el marco de incrementos cercanos al $5 \%$ en el parque móvil y al 3\% en el censo de conductores. ¡Los accidentes de circulación pueden ser evitados mediante políticas de seguridad vial adecuadas!

El volumen de negocio vinculado al tráfico y el transporte convierte a este sector en parte esencial del engranaje de la vida económica de los países industrializados... y de los que aspiran a serlo. 
De hecho, la industria del automóvil da empleo en España a casi el veinte por ciento de la población activa. Pero, también es parte esencial de ese engranaje el costo económico atribuible a los accidentes de circulación. Ciertamente, las tasas de letalidad están disminuyendo en los llamados paises ricos, pero aumentan con rapidez en el mundo en desarrollo, y muy especialmente en Asia, donde el número de defunciones imputables al tráfico rodado está incrementándose como promedio en torno al $90 \%$. El problema es tan grave que, de aquí al año 2020, los traumatismos por accidentes de tráfico probablemente pasarán a constituir a nivel mundial la segunda causa más importante de pérdida de años potenciales de vida.

Además de los costos directos asociados a los traumatismos y las muertes en carretera, el brutal aumento en el número de vehículos y el mantenimiento de determinadas politicas de transporte tienen también otras repercusiones sanitarias muy graves, así como efectos sociales, económicos y ambientales más amplios. En algunos países, la contaminación atmosférica achacable a las emisiones atribuibles al transporte por carretera causa aún más víctimas mortales que los propios accidentes de circulación. Además de los efectos directos que tiene en las enfermedades respiratorias y coronarias, el transporte motorizado produce aproximadamente la cuarta parte de las emisiones antropógenas de gases responsables del cambio climático. Lamentablemente esas "epidemias ocultas" reciben, pese a su importancia diferencial, una atención nacional o internacional relativamente escasa en comparación con la prestada a las principales enfermedades transmisibles y no transmisibles.

Como contrapunto, el costo de los accidentes de circulación supera el $2 \%$ del PIB, similar que en todos los países desarrollados (Organización Mundial de la Salud, 2004), si tenemos en cuenta todos los efectos derivados de los mismos, como bajas/incapacidades laborales, absentismo, pérdida de productividad, asistencia sanitaria, reparación de daños materiales, tramitaciones, peritajes, o la atención de todo tipo de secuelas (pensiones por invalidez, orfandad o viudedad, etc.). El que hablemos de un mal común que acontece en otros países desarrollados y masivamente motorizados tanto de nuestro continente, como de otros, no debe servirnos de consuelo, sino de acicate.

Pese a las directivas europeas que han venido apareciendo, lo cierto es que cada país, aún compartiendo los grandes objetivos (por ejemplo, reducir en un $50 \%$ el número de accidentes y de muertes en la UE, fuente: CARE -EU road accidents database), concreta con grados de libertad sus propias politicas de prevención. Así, en Europa coexisten diversos modelos (Barjonet y Tortosa, 2001; Tortosa, Barjonet, Civera y Montoro, 2003), siendo, sin duda, el que más potencial preventivo tiene, y más historia (Serra y Tortosa, 2003), el español.

Frente a aquellos que consideran el accidente como un suceso fruto del destino, imprevisto y fortuito, que escapa de nuestro control, que depende de los otros, que es inevitable... los especialistas consideran que es un problema más de salud, por tanto consecuencia de un proceso, prevenible, evitable y controlable (Montoro, Alonso, Esteban y Toledo, 2000, Montoro, Toledo, Lijarcio y Roca, 2006). Una opinión compartida por lo demás por los conductores españoles, quienes consideran que aproximadamente el $90 \%$ de los accidentes de tráfico son debidos al genéricamente llamado "factor humano" (Civera, Pastor y Alonso, 2003; Montoro, Toledo, Lijarcio y Roca, 2006). 


\section{UNA ESCUETAS NOTAS HISTÓRICAS SOBRE EL MARIDAJE ENTRE PSICOLOGÍA Y SEGURIDAD VIAL}

Durante el primer tercio del siglo XX la respuesta legal a la prevención de accidentes de circulación comenzó su camino hacia una regulación jurídica propia y autónoma, si bien parte de ella todavía se repartiria entre los Reglamentos de Policía y Conservación de las Carreteras y los Reglamentos Municipales de Circulación. La historia del proceso (Cano, 2001, 2002) hacia una legislación autónoma comienza con la R.O. de 31-VII-1897 que exigía el permiso de circulación sobre los nuevos vehículos de tracción no animal, y con el más amplio Reglamento para el Servicio de Coches Automóviles ("todos los carruajes movidos por fuerza mecánica") por las Carreteras del Estado de 1900, y encontrará como principal jalón de referencia el Código de Circulación de 1934.

El antecedente más inmediato de aquel Código lo encontramos, en plena edad de oro de la psicotecnia (Gundlach, 1998; Capdevila y Saiz, 1998), en la ciudad de Barcelona, "la primera ciudad del mundo que implantó el examen psicotécnico en la circulación urbana en toda su extensión y con la fuerza coercitiva del dictamen psicotécnico" (Giese, 1933, 155). Efectivamente, el Ayuntamiento de Barcelona incluyó en la concesión de un servicio urbano de autobuses, la obligatoriedad de que sus conductores obtuviesen un certificado de aptitud profesional: "El concesionario vendrá obligado a someter a los conductores de sus vehículos a un reconocimiento previo de aptitud psicofisiológico en el Instituto de Orientación Profesional, en cuyo centro se les expedirá gratuitamente el oportuno certificado de aptitud, sin el cual no podrán entrar al servicio de la empresa, bajo la responsabilidad de esta" (Granada, 1929, 29).
Mira (Saiz y Saiz, 1996) estableció unas pruebas de aptitud que definían un plan de examen "más que suficiente para hacer una selección práctica de los conductores buenos, normales y malos" (Mira, 1922-1923/1989, 40), con lo que de facto definía un modelo de examen que, con pocas variaciones en su fondo (Ozcoidi, 2001; Tortosa y Montoro, 2002), sigue vigente: "Descartado el examen de las condiciones sensoriales (hecho en el laboratorio médico) nos ha parecido que los puntos esenciales que nos era necesario investigar eran los siguientes: percepción de velocidades y distancias, distribución, intensidad y duración de la atención visual, rapidez y precisión de las reacciones oculomotríces $\mathrm{y}$ audiomotrices, $\mathrm{y}$, finalmente, grado $\mathrm{y}$ tipo emotivo del sujeto examinado (...) la parte más esencial del trabajo de conducción de vehículos no es la parte que podríamos denominar de reacción sino la parte de percepción, incluyendo en ella los actos de asociación de los estímulos percibidos, según determinadas circunstancias y la adopción del movimiento a hacer (es decir, lo que podríamos denominar la parte judicativa del trabajo)" (Mira, 1922-1923/1989, 40). Esos exámenes no sólo suministraban un diagnóstico de las aptitudes, sino que permitian formular un pronóstico acerca de la actuación futura del aspirante, dimensión que cumplía muy satisfactoriamente el examen (Soler-Dopff, 1930).

Se había definido un campo y rol de actuación profesional, la (entonces) Psicotecnia aplicada al tráfico y el transporte de personas y mercancías (tranvías, trenes, autobuses, camiones, coches, aviones, barcos). Un rol que, no sólo se ha mantenido, sino que se ha venido ampliando (y legislando) dentro de la (hoy) llamada "Psicología de la Seguridad (p.e., Vial, Aeronáutica, Ferroviaria, Naval)", ayudando a conformar el hoy denominado "modelo español" de Seguri- 
dad Vial (Tortosa y Montoro, 2002). Un modelo en el que, desde su nacimiento, los aspectos físicos necesarios para un adecuado desempeño de la tarea de conducción los exploraban y evaluaban los profesionales de la Medicina; mientras que los aspectos (normales y patológicos) psicológico-aptitudinales eran explorados por profesionales de la Psicotecnia primero, y de la Psicología después.

El objetivo de la exploración era, y es, realizar, individualmente, una valoración del estado psicofísico de cada aspirante, con arreglo a unos parámetros de normalidad (física y psiquica), obtenidos mediante la utilización de instrumentos y pruebas homologados. Con ello se pretende más que predecir el mayor 0 menor riesgo de un potencial conductor de sufrir o producir conflictos o accidentes, establecer (o prever) el nivel de deterioro, o de posible deterioro, de dimensiones psicofísicas relevantes para un adecuado desempeño como conductor, en el convencimiento de la posible influencia de dicho estado sobre la calidad de la conducción.

La historia, desde entonces hasta hoy, así como su extensión a Iberoamérica (Hoffmann, Tortosa y Carbonell, 1994), la hemos contado ya (Civera, Pastor y Alonso, 2003; Montoro, Carbonell y Tortosa, 1991; Serra y Tortosa 2003; Soler y Tortosa, 1987; Tortosa, Civera y Pastor, 2001), y no vamos a abundar en ella (ver figura 1 para principales jalones y extensiones).

\section{FUNCIONAMIENTO ACTUAL}

En la historia a la que haciamos referencia, destaca como otro momento clave la extensión de la exploración desde centros públicos donde se exploraban conductores profesionales, a centros priva- dos donde exploran a TODOS los que aspiran a obtener y/o renovar los carnés de conducir, y lo hacen, como antes, con una cierta periodicidad dado que las destrezas y el estado de salud, física y mental, puede cambiar con el tiempo.

Además de ello, es importante destacar una reciente novedad surgida como consecuencia de la implantación en España del llamado sistema de permiso y licencia de conducción por puntos. Como es bien sabido, mediante este sistema aquellos infractores multireincidentes que demuestren tener unos hábitos de conducción totalmente incompatibles con las normas más básicas de seguridad vial perderán la vigencia de su permiso de conducir y, por tanto, serán apartados de la circulación vial hasta que cumplan con una serie de requisitos legales establecidos para ello. Estos requisitos incluyen un tiempo minimo de penalización antes de poder recuperar el permiso o la licencia (en general, seis meses), la asistencia y el aprovechamiento de un curso formativo de sensibilización y reeducación vial, aprobar un examen sobre dicho curso y, finalmente, la obtención del correspondiente certificado de aptitud en un Centro de Reconocimiento de Conductores (Ley 17/2005, de 2/VII).

Este hecho es importante para la Psicologia del Seguridad Vial por varios motivos. En primer lugar, constituye una oportunidad para los profesionales de la psicologia que trabajan en los Centros de Reconocimiento para tomar contacto directo con un colectivo de conductores cuyo problema al volante va, probablemente, más allá de lo psicofísico y se adentra en áreas psicológicas tan relevantes para la seguridad vial, como son las actitudes, la personalidad o los trastornos adictivos, por poner algunos ejemplos. Ello, aunque a día de hoy no se haya abordado de forma directa, 


\section{Figura 1. Cronograma del desarrollo legislativo}

\begin{tabular}{|c|c|}
\hline $\begin{array}{l}\text { R.D. 1467/1982, de } 28 N \text {, } \\
\text { describe enfermedades y } \\
\text { deficiencias que impiden la } \\
\text { obtención o revisión de los } \\
\text { permisos de conducción y } \\
\text { condiciones de certificados y } \\
\text { reconocimientos (desarrollado por } \\
\text { O.M. de 22/9/82) }\end{array}$ & \\
\hline $\begin{array}{l}\text { R.D. 2272/1985, d e } 4 / X I I \text {, que } \\
\text { determina las aptitudes } \\
\text { psicofisicas de los conductores de } \\
\text { vehiculos y regula los centros de } \\
\text { reconocimientos destinados a } \\
\text { verificarlas. }\end{array}$ & $\begin{array}{l}\text { R.D. 2283/1985, de } 4 / X I I \text {, por } \\
\text { el que se regula la emisión de } \\
\text { los informes de aptitud } \\
\text { necesarios para la obtención } \\
\text { de licencias, permisos y } \\
\text { tarjetas de a rmas, modificado } \\
\text { por } 0 . \mathrm{M} \text {. de } 4 / 1 / 2000 \text {, por la } \\
\text { que se varia el anexo } 2 \\
\text { relativo a las tarifas aplicables } \\
\text { a los informes de aptitud. }\end{array}$ \\
\hline $\begin{array}{l}\text { R.D. 1342/1986, de } 26 N \text {, que } \\
\text { modifica los arts } 6^{\circ}, 2^{\circ} \text { y } 13^{\circ} \text { del } \\
\text { R. D. 2272/85, atribuyendo a } \\
\text { Sanidad las competencias } \\
\text { dirimentes en materia de } \\
\text { discrepancias psicológicas. } \\
\text { Especifica la autorización de } \\
\text { apertura de centros en materia } \\
\text { sanitaria. }\end{array}$ & \\
\hline $\begin{array}{l}\text { Resolución DGT de III/1987. } \\
\text { Normalización de instrumentos y } \\
\text { materiales utilizados por los CRC } \\
\text { en las exploraciones } \\
\text { psicotécnicas }\end{array}$ & \\
\hline $\begin{array}{l}\text { - La Directiva 91/439/CEE, de } \\
\text { 29NII/1991, sustituye a la primera } \\
\text { directiva del Consejo de } 1980 \text {. }\end{array}$ & \\
\hline & $\begin{array}{l}\text { - Ley } 23 / 1992 \text { de } 30 / \mathrm{VII} \text {, de } \\
\text { Seguridad Privada. Regula las } \\
\text { condiciones a superar para } \\
\text { prestar servicios como } \\
\text { vigilantes de seguridad y } \\
\text { guardas particulares del } \\
\text { campo. }\end{array}$ \\
\hline & $\begin{array}{l}\text { R.D. } 137 / 1993 \text {, de } 291 \text {, por el } \\
\text { que se aprueba el Reglamento } \\
\text { de Arm as, modificado por el } \\
\text { R,D, } 540 / 1994 \text {, de } 25 / 11 \text { y por } \\
\text { el R,D. } 316 / 2000 \text {, de } 3 / I I \text {. Su } \\
\text { articulo } 98 \text { regulaba la emisión } \\
\text { de los informes de aptitud } \\
\text { necesarios para la obtención } \\
\text { de licencias, permisos y } \\
\text { tarjetas de armas. }\end{array}$ \\
\hline & $\begin{array}{l}\text { R.D. 2364/1994, d e 9/XII. } \\
\text { Aprueba el Reglamento de } \\
\text { Segunidad Privada. Establece } \\
\text { la necesidad de evaluar las } \\
\text { aptitudes fisicas y psiquicas } \\
\text { necesarias para ejercer sus } \\
\text { funciones. }\end{array}$ \\
\hline
\end{tabular}


Figura 1. Cronograma del desarrollo legislativo (continuación)

\begin{tabular}{|c|c|c|c|}
\hline$\bullet$ & $\begin{array}{l}\text { R.D, } 772197 \text { de } 30 / N \text {. Reglamento } \\
\text { General de Conductores } \\
\text { (Modificado por el R.D. } 1598 / 2004 \\
\text { de } 2 N \text { III). En su Anexo IV, recoge } \\
\text { "Las aptitudes Psicofisicas para } \\
\text { obtener y prorrogar el permiso o } \\
\text { liœencia de conducción } \\
\text { (modficado por R.D. } 1598 / 2004 \\
\text { de } 2 N \text { NII) }\end{array}$ & & $\begin{array}{lr}\text { O.M. de } & 17 / \mathrm{N} / 1997, \\
\text { desarrollada } & \text { por } \\
\text { Resolución r de } & \text { do/XII/1997. Acreditación } \\
\text { de la aptitud psicofisica } \\
\text { para obtener o renovar las } \\
\text { titulaciones para el } \\
\text { gobierno } \\
\text { embarcaciones de recreo } \\
\end{array}$ \\
\hline & & $\begin{array}{l}\text { - R.D. } 2487 / 1998 \text {, de } 20 / X I \text {, por } \\
\text { el que se regula la } \\
\text { acreditación de la aptitud } \\
\text { psicofisica necesaria para } \\
\text { tener y usar armas y para } \\
\text { prestar servicios de seguridad } \\
\text { privada. }\end{array}$ & \\
\hline & & $\begin{array}{l}\text { - O.M. de 14/l/1999, por la que } \\
\text { se aprueban los modelos de } \\
\text { informes de aptitud psicofisica } \\
\text { necesaria para tener y usar } \\
\text { armas y para prestar servicios } \\
\text { de seguridad privada. }\end{array}$ & $\begin{array}{l}\text { - Ley } 50 / 1999 \text { de } 23 / X I I . \\
\text { Acreditación de la aptitud } \\
\text { psicofisica para tenencia } \\
\text { deranimales } \\
\text { potencialmente peligrosos } \\
\text { (R,D. 287/2002 de 22/III) }\end{array}$ \\
\hline & & $\begin{array}{l}\text { - O.M. 904/2002, de } 15 / \mathrm{V} \text {, de } \\
\text { normalización de impresos de } \\
\text { solicitud de licencias de armas } \\
\text { de fuego y otras } \\
\text { autorizaciones. }\end{array}$ & $\begin{array}{l}\text { - R.D. 270/2000 de 25/II. } \\
\text { Determina las condiciones } \\
\text { para el ejercicio de las } \\
\text { funciones del personal de } \\
\text { vuelo de las aeronaves } \\
\text { civiles desarrollado por la } \\
\text { O.M. de 21/1l/2000 }\end{array}$ \\
\hline & $\begin{array}{l}\text { Ley } 1712005 \text {, de } 2 \mathrm{NII} \text {, por la que } \\
\text { se regula el permiso y la licencia } \\
\text { de conducción por puntos y } æ e \\
\text { modifica el texto articulado de la } \\
\text { ley sobre tráfico, circulación de } \\
\text { vehiculos a motor ys eguridad } \\
\text { vial. }\end{array}$ & & $\begin{array}{l}\text { - R.D. } 836 \text { y } 83712003 \text { de } \\
27 N \text {. Acreditación de la } \\
\text { aptitud psicofisica para } \\
\text { operador de g rúa torre- } \\
\text { grúa móvil }\end{array}$ \\
\hline & $\begin{array}{l}\text { RESOLUCIón de } 26 \text { de agosto } \\
\text { de } 2007 \text {, de la Dirección General } \\
\text { de Tráfico, por la que se crea un } \\
\text { registro clectrónico cn la J cfatura } \\
\text { Central de Tráfico. }\end{array}$ & & \\
\hline
\end{tabular}

podría hacer recomendable un estudio científico y riguroso, que permita identificar qué complementos deberían incorporarse al protocolo de actuación de estos profesionales, de forma que se establezca un procedimiento más especializado y más adecuado al perfil y a las particulares circunstancias de estos conductores.
En segundo lugar, el permiso por puntos también ha contado con la participación de psicólogos con conocimientos específicos en tráfico y seguridad vial (provenientes en su mayoria de Centros de Reconocimiento) para el desarrollo de una sesión de dinámica de grupos de dos horas de duración dentro de los cursos de sensibilización y reeducación a los 
que haciamos referencia anteriormente. Este papel, aunque pequeño en comparación, por ejemplo, con las horas concedidas a los formadores que deben impartir dicho curso (no se debe olvidar que el curso persigue un objetivo meramente formativo, dirigido a promover el debate y la reflexión sobre cuestiones básicas de seguridad vial), constituye también una oportunidad para conocer las especiales circunstancias de este colectivo de conductores y definir el futuro rol que podría tener la psicología dentro del esquema de reeducación de conductores multireincidentes.

La normativa española actual cumple, además, los requisitos reflejados en las diferentes Directivas Comunitarias, tanto en cuanto a los considerados como requisitos rígidos, como en los que, dada su flexibilidad, son llamados requisitos de mínimos. La unidad básica de este modelo son los Centros de Reconocimiento Médico y Psicotécnico, que tienen como objetivo la evaluación de las condiciones de aptitud psicofísica, para determinar la capacidad de los conductores y, según ello, establecer en caso necesario las restricciones, adaptaciones o limitaciones que permitan unas condiciones de conducción más seguras (Mirabet y otros, 1991; Montoro y Mirabet, 2003). Por la red estatal de Centros de Reconocimiento Médico y Psicotécnico pasan anualmente más de 2 millones de conductores con el objeto de obtener, prorrogar y -desde el permiso por puntos- recuperar algún tipo de permiso de conducir. Estas personas son reconocidas por un equipo de facultativos, los cuales emitirán un informe basándose en la normativa vigente.

La valoración médico-psicológica se lleva a cabo siguiendo los 13 apartados del Anexo legal regulador de las aptitudes psicofisicas requeridas para obtener o prorrogar el permiso o la licencia de conducción (R. D. 1598/2004, de 2 de julio). Establece las enfermedades y deficiencias que serán causa de denegación o de adaptaciones, restricciones de circulación y otras limitaciones en la obtención o prórroga de cualquier clase de permiso o licencia de conducción. En cada uno de estos apartados se especifica la exploración que debe realizarse, los niveles mínimos de aptitud, las condiciones restrictivas en cada una de las situaciones y también la necesidad, por parte del conductor, de aportar informes específicos en determinados casos. En la base de datos (ver RESOLUCIÓN de 26 de agosto de 2007, de la Dirección General de Tráfico) se deben incluir datos de identificación; datos de carácter general (edad, sexo, variables sociales y de salud); variables que recogen las condiciones psicofísicas que deben reunir los conductores (Anexo IV), los resultados de las diferentes pruebas realizadas, y el resultado final del informe emitido y las restricciones (limitaciones y adaptaciones) en caso de ser necesarias.

El protocolo de actuación de los profesionales es claro. Estamos hablando de una intervención profesional en la que pueden distinguirse dos niveles de actuación, uno básico y otro específico. En este punto, nadie debe olvidar que la exploración básica sigue las directrices que distinguen a los métodos de despistaje o screening, ya que se aplica a muchas personas, su protocolo de aplicación descansa sobre una ejecución sencilla y ágil, con un costo económico libre (pero bajo), y reproducible, con los profesionales y el instrumental apropiado, en cualquier lugar y momento.

Si ninguno de los profesionales encuentra indicio alguno de patologías o deficiencias restrictivas en ese primer nivel del reconocimiento, se podrá emitir el informe definitivo, en este caso de "Apto". Si, por el contrario, cualquiera de 
los profesionales detecta o descubre indicios que hacen sospechas la "simulación”, o la presencia de patologias o deficiencias que puedan limitar la capacidad de conducir, se deberá pasar a un segundo nivel de intervención, una exploración específica. En este caso, la evaluación es más compleja y profunda, incluso en ocasiones resultará preciso recurrir a fuentes de información externas al Centro de Reconocimiento Médico y Psicotécnico, como son atención primaria (médico de cabecera), especialista (si esta bajo tratamiento de una unidad específica)... Los resultados de la evaluación específica, junto con los datos externos si se hubieren requerido, permitirán al profesional o profesionales realizar una nueva valoración, y en base a ella emitir un informe final. Un informe que, según la normativa vigente, podrá ser de "Apto", "Apto con algún tipo de restricción, limitación o adaptación"; o de "No Apto" (Figura 2).

Figura 2. Organigrama del proceso de exploración

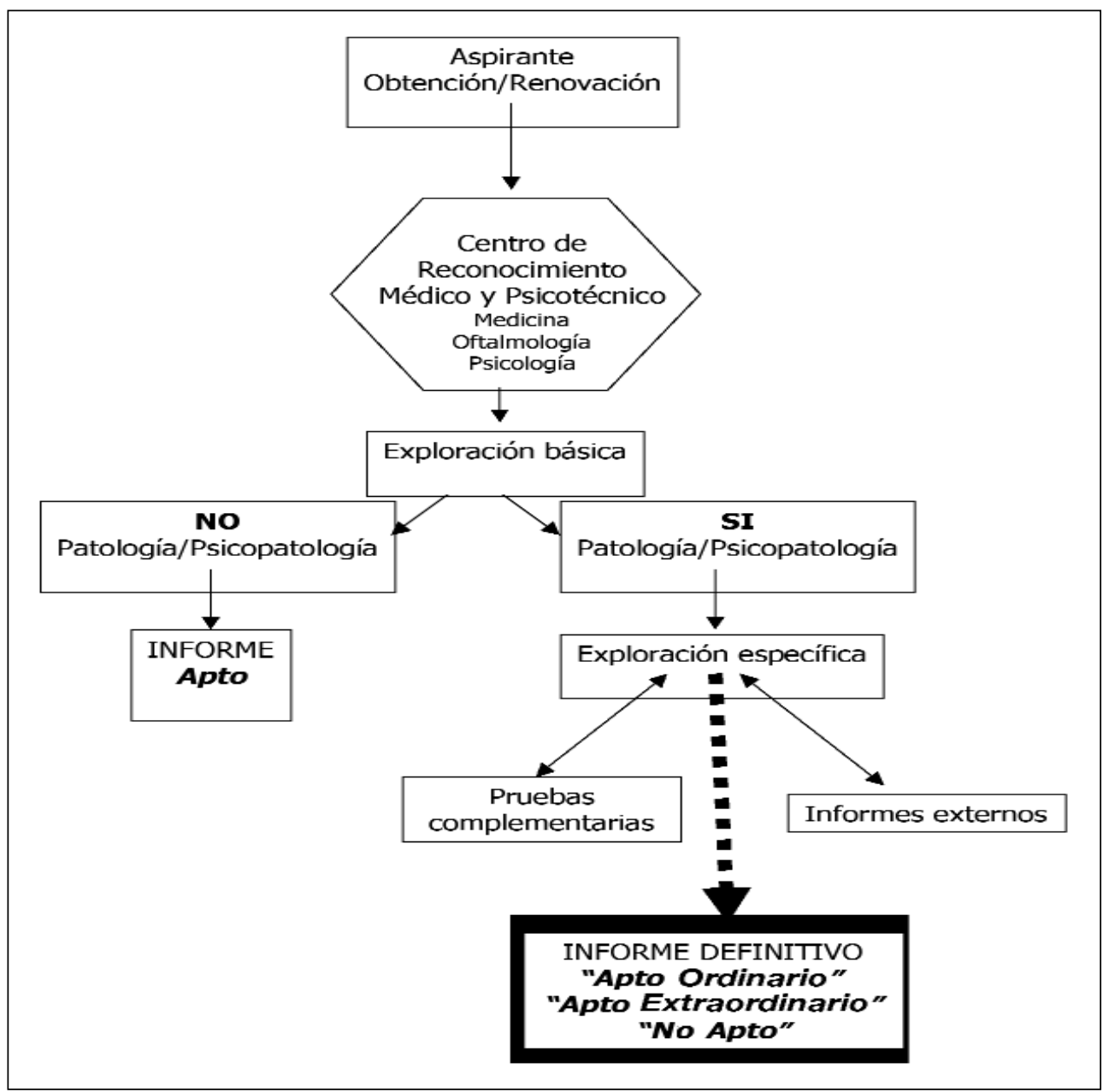


Sin entrar en casos concretos, denunciados por la Administración a partir de su legítima acción de inspecciôn, creemos que hay razones de peso, aparte de la propia expresión de satisfacción de los usuarios respecto al conocimiento de problemas de salud que desconocían (Serra, 2003), que avalan el modelo. Entre ellas las siguientes:

- Más de 100 años de ciencia y práctica psicológica profesional rigurosa y eficaz, tanto fuera de España (Gundlach, 1998; Tortosa, Barjonet, Civera y Montoro 2003), como dentro (Civera, Pastor y Alonso, 2003; Tortosa, Quintanilla, Civera y Díaz, 1993; Santolaya, Berduyas y Fernández-Hermida, 2002).

- Casi 100 años de existencia como modelo, superando todos los modos posibles de gobierno - dictablandas y dictaduras; monarquías de diversa indole, regimenes autoritarios y democráticos, e incluso un fallido intento republicano- (Serra, 2003; Ozcoidi, 2004; Terrrassa, 2005).

- Subyace a las actuaciones profesionales una buena formación curricular de base. Los Planes de Estudios habilitan para un riguroso ejercicio profesional. Un ejercicio profesional inspirado desde modelos cognitivo-conductuales, eclécticos y conductuales (Santolaya, Berdullas y Fernández-Hermida, 2002), dominantes en el currículo formativo (Civera, Santolaya y Tortosa, 2006).

- Existen ya perfiles profesionales social y laboralmente reconocidos, el de "Psicólogo del Tráfico y de la Seguridad" (COP, 1998, 140) y el de "Médico del Tráfico", ambos, además, con oferta formativa y de reciclaje específica, provenientes de la iniciativa pública y privada, y de los propios Colegios Profesionales.

- En las revisiones aparece una destacable investigación (no siempre conocida y/o tenida en cuenta) científica y (psico)tecnológica en Seguridad Vial, tanto sobre aspectos de la intervención preventiva, como en ámbitos de investigación de I, I+D, e I+D+I (Prieto, Fernández-Ballesteros y Carpintero, 1994; Fernández, 2002a, 2002b).

- Las magnitudes del complejo fenómeno del tráfico y el transporte, y sus secuelas, definen un problema de salud pública que rebasa el límite de acción de grupos e individuos (Montoro, Toledo, Lijarcio y Roca, 2006). Como tal exige un modelo sanitario como el vigente, que va desde la educación para la salud, la prevención primordial, hacia una cultura de la seguridad y la salud.

No obstante, el criterio más definitivo en ciencia es, sin duda, la eficacia y a ello vamos a dedicar el siguiente apartado.

\section{¿LOS CENTROS DE RECONOCIMIENTO MÉDICO Y PSICOTÉCNICO DETECTAN PROBLEMAS DE SALUD?}

La evolución de la normativa no ha hecho sino ir potenciando el papel de los Centros de Reconocimiento como unidades preventivas de problemas de salud, ya que anualmente chequean a cerca de tres millones de españolas y españoles que aspiran a realizar diferentes conductas que entrañan riesgos. Es más, la obligada periodicidad de la renovación de los permisos y licencias, lleva a que toda la población que ha tenido o tiene en vigor (además de a los que a ello han aspirado) un permiso de conducir $\mathrm{y} / \mathrm{o}$, 
por ejemplo, una licencia de armas, haya debido efectuar un reconocimiento en alguno de los más de 2.000 Centros existentes.

Hipotéticamente hablando, esa presumible acción preventiva les habrá llevado a contribuir en el nivel de bienestar social general mediante la detección de problemas de salud en muchos casos desconocidos por quienes acuden a los Centros de Reconocimiento Médico y Psicotécnico, pero ¿realmente es asi?

No existe una abundante bibliografia al respecto, pero la existente parece indicar que es así, se contribuye a la prevención, y además los usuarios lo reconocen (ver Serra, 2003; Ozcoidi, 2004; Terrassa, 2005). En cualquier caso, nosotros hemos pretendido contribuir a esa bibliografía y en una amplia muestra de personas mayores de 55 años hemos realizado un estudio empírico ${ }^{1}$.

Las razones de la elección, dejando aparte el hecho de que los estudios epidemiológicos les ubican como grupo de riesgo y que los datos demográficos muestran el crecimiento continuado de esta población, radican en el hecho de que se trata de un grupo vulnerable a los problemas de salud, y muy vulnerable, especialmente como peatones, a problemas de tráfico (Alonso, Fernández, Esteban, Calatayud, Tortosa, Monteagudo, Alamar, Chofre y Montoro, 2004). Sufren, con los años, un deterioro de sus dimensiones psicofísicas que, presumiblemente (Montoro y Mirabet, 2003), debe ser detectable en las exploraciones psicofísicas de los Centros de Reconocimiento Médico y Psicotécnico. Efectivamente, van experimentando limitaciones funcionales en sus capacidades psicomo- toras y en las sensoriales (especialmente en la visión y la audición), son más vulnerables a factores externos de la conducción y tienen una mayor vida interior, sufren más dolencias físicas $\mathrm{y}$, consecuentemente, muestran unos niveles elevados de consumo de medicamentos, incluso de policonsumo (riesgo elevado de "efectos laterales sinérgicos"), y lo que es más grave, muestran las mayores tasas de automedicación.

Hemos accedido a una muestra de 28.083 personas aspirantes a la obtención o renovación del carné de conducir en centros de diferentes comunidades autónomas, explorados en 2006. El grupo diana lo definian 7.517 conductoras y conductores mayores de 55 años, un $27 \%$ de las personas exploradas. De ellos 3.648, 49\% de la muestra de mayores y $13 \%$ de la muestra total, tenian entre 55 y 69 años; y 3.869, 51\% de la muestra de mayores y $14 \%$ de la muestra total, tenian más de 70 años.

Sólo un 35\% de las personas mayores obtuvieron el informe de "Aptos", sin condiciones. En un 65\% de los casos se detectaron problemas de salud, que obligaron a lentes $\mathrm{y} / 0$ audífonos, 0 a introducir adaptaciones, restricciones y/o limitaciones, e incluso a acudir a especialistas, en tres de cada cinco personas exploradas. Una de cada 100 personas exploradas fue declarada "No apta" por razones diversas, y ocho de cada cien obtuvieron un resultado de "Interrumpido"

Los resultados en población general cambian substancialmente, Así, en los menores de 55 años, más del $60 \%$ obtienen el informe de "Aptos" sin condiciones. Uno de cada ocho mil es considera-

\footnotetext{
${ }^{1}$ Agradecemos a la CREME (Federación de Asociaciones de Centros de Reconocimientos Médicos) la cesión de datos, y muy especialmente a Marta Ozcoidi, Bartolomé Terrasa, Africa Vicondoa y Joan Serra.
} 
do "No Apto", y aproximadamente dos de cada cien se verán obligados a introducir adaptaciones, restricciones y/o limitaciones, e incluso a acudir a especialistas. El porcentaje de "Aptos Ordinarios (Lentes y/o Audifonos)" es prácticamente la mitad entre los menores de 55 años que en los grupos mayores de edad.

La mayor prevalencia desde una perspectiva médica se encontró en deficiencias sensoriales en la visión (agudeza visual, visión monocular, afaquias) y audición (agudeza auditiva): alteraciones en el sistema locomotor y muscular (trastornos de movilidad, tono muscular y coordinación); trastornos neurológicos; afecciones del aparato respiratorio y circulatorio; enfermedades cardiovasculares (sobre todo alteraciones en el ritmo y coronariopatias); enfermedades metabólicas (diabetes); trastornos mentales y de conducta; y, sobre todo en la aptitud perceptivo-motora (cuatro veces más que en los más jóvenes), donde son dominantes los problemas en coordinación bimanual. En el caso de los ámbitos de competencia del psicólogo fueron los trastornos por substancias (sobre todo por consumo excesivo de alcohol), los deterioros cognitivos y psicomotores, los trastornos de ansiedad y otros trastornos de conducta y de la personalidad. En cuanto a las condiciones restrictivas las más habituales, en justa correspondencia, han sido: corrección de la visión, prótesis auditivas, limitación conducción diurna, limitación conducción nocturna, limitación radio kilómetros, limitación de velocidad, conducción no permitida en autopista, adaptaciones en el vehículo (transmisión, embrague, mecanismo frenado, mecanismo aceleración, dirección, asiento del conductor), periodo de vigencia.

En definitiva, cumplen con su cometido, previenen, detectan problemas de salud desconocidos en muchos casos para quienes los padecian, y por tanto mejoran los niveles de salud de la población general. Además, se están ofreciendo valoraciones de "No Apto" con lo que se aparta de la conducción a personas que no están capacitadas para conducir con seguridad. Cumplen, pues, satisfactoriamente, sus objetivos, que no son los de apartar de la conducción a muchos y muchas, sino sólo a quienes, por razones diversas, no están en disposición de controlar adecuadamente esa actividad de riesgo. Insistimos en ello, el objetivo de la normativa no es impedir, sino conseguir que quienes conduzcan utilicen su carné con prudencia, responsabilidad y control... Para ello establece una acción evaluadora sobre ciertos requerimientos: condiciones físicas, destrezas psicomotoras, adecuado nivel intelectual, equilibrio y uso racional de todo ello por un psiquismo sin factores disposicionales adversos...

Una acción evaluadora siempre dificil, ya que el ser humano no es un organismo pasivo que emite respuestas automáticas ante estimulos de un entorno, sino que es una persona, que construye sus situaciones y las dota de sentido, valor y niveles de riesgo, y que para conseguir sus objetivos es capaz incluso de mentir y/o simular. Una acción evaluadora para la que los psicólogos están adecuadamente formados.

En sintesis, la estrategia fundamental de prevención desde los Centros de Reconocimiento Médico y Psicotécnico se centra, por una parte, en la eliminación de los factores de riesgo en el tráfico, identificando personas con patologias o deterioros que pudiesen repercutir negativamente sobre las competencias que permiten conducir con seguridad. Por otra parte, se orienta hacia la educación psico-socio-sanitaria y vial, con objeto, desde modelos de creencias de salud, de transmitir información para producir cambios en las actitudes y comporta- 
mientos de quienes conducen. La acción educadora debe ser pluridisciplinar e intersectorial, dirigida a promover cambios en las personas, fomentando los recursos individuales, para incrementar la posibilidad de elegir opciones saludables y/o seguras (Alonso, Esteban, Calatayud, Tortosa, Monteagudo, Alamar, Montoro y Chofre, 2004; Ozcoidi, Sanz, Reula y Gonzalez Luque, 2002).

\section{BIBLIOGRAFÍA}

Alonso,F., Fernández, C; Esteban, C.; Calatayud, C.; Tortosa, F.; Monteagudo, M.J.; Alamar,B.; Chofre,E. y Montoro,L. (2004). Ancianos de hoy en tráfico. Barcelona: Iniciativas de Educación y Seguridad Vial.

Alonso, F.; Esteban, C.; Calatayud, C.; Tortosa F.; Monteagudo, M.J.; Alamar, B.; Montoro, L.; Chofre, E. (2004). Programa de educación vial para personas mayores. Barcelona: Iniciativas de Educación y Seguridad Vial.

Barjonet, P. y Tortosa, F. (2001). Transport Psychology in Europe: A Historical Approach. En P. Barjonet (Ed.): Traffic Psychology Today (pp 1329). A,H, Dordrecht. The Netherlands. Kluwer Academic Publishers

Cano, T. (2001). El permiso de conducción en España: significado y régimen jurídico. En Dirección General de Tráfico (Ed.): 100 años del Permiso de Conducción en España. Recopilación de trabajos Premiados (55-62), tomo I, Dirección General de Tráfico. Madrid.

Cano, T. (2002). El permiso de conducción en España: significado y régimen jurídico. Ed. Civitas.

Capdevila, A. y Sáiz, M. (1998) El inicio del estudio del factor humano en la conducción de transportes en Cataluña. Revista de Psicología del Trabajo y de las Organizaciones, 14(2), 233250.

Civera,C., Pastor, J.C. y Alonso,F. (2003). Un siglo de selección de conductores en España. Revista Mexicana de Psicología: 20-(2), 301-313.

Civera, C. Santolaya, F. y Tortosa, F. (2006). Psicologia y profesión en la España contemporánea. En F.Tortosa y C.Civera, Historia de la Psicología (pp. 451-469). Madrid: McGraw-Hill.

Colegio Oficial de Psicólogos (1998). Perfiles profesionales del psicólogo. Madrid. Colegio Oficial de Psicólogos.
Creme Federación. Federación de Asociaciones de Centros de Reconocimientos Médicos.

Fernández,J. (Ed.) (2002a): Líneas de investigación en la psicología española (19891998). 1ํㅡㄹ Parte. Papeles del Psicólogo, Época III, $\mathrm{n} \div 81$.

Fernández,J. (Ed.) (2002b): Líneas de investigación en la psicologia española (1989-1998). $2^{\text {a }}$ Parte. Papeles del Psicólogo, Época III, noำ.

Giese, F. (1933). Psicotecnia. Barcelona: Labor.

Granada, A. (1929). La selección profesional de los chóferes. Análes de la Sección de Orientación Profesional de la Escuela de Trabajo, Marzo, II(2), 29-34

Gundlach,H. (1998). El desarrollo de la psicología aplicada en Europa Central. En F. Tortosa (Coord.), Una Historia de la Psicología Moderna (pp 389-397). Madrid: McGraw Hill.

Hoffmann, M.H, Tortosa, F. y Carbonell, E. (1994). Emilio Mira y López y el desarrollo de la psicología del transito. Los casos de España y Brasil. Revista Latinoamericana de Psicología. 26(3), 495-516.

Hoyos,C. (1992). A changed in perspective. Safety Psychology replaces the traditional field of accident research. The German Journal of Psychology, 16(1), 1-23.

Mira, E. (1922/1923/1989). La selecció dels xófers de la companya general d' autòmnibus. Anals de l' Institut d' Orientació Professional, Any III i IV (6-7), 60-71. En F. Tortosa, L. Montoro y E. Carbonell, Psicología y Tráfico en España: 60 años de historia. Zaragoza: Librería General.

Mirabet E, Garcia P, Pertejo E, De Vega P y Requena, E. (1991). Los centros de reconocimiento médico y psicotécnico en la prevención de accidentes de tráfico: 1480 reconocimientos realizados en 
el centro de Cruz Roja Española (Valencia). Revista de Sanidad e Higiene Pública; 65: 165-73.

Montoro, L. ; Carbonell, E. y Tortosa, F. (1991). Psicología y Seguridad Vial en España. 70 años de historia. Revista de Historia de la Psicología, 12(2), 157-174.

Montoro, L.; Alonso, F.; Esteban, C. y Toledo, F (2000). Manual de Seguridad Vial: El factor humano. Barcelona: Ariel-INTRAS.

Montoro, L. y Mirabet, E. (1991). El modelo español de reconocimiento médico y psicotécnico en el contexto de la $2^{\text {a }}$ Directiva (91/439/CEE). Resultados al ser aplicado sobre un grupo especifico de conductores (45 a 70 años). Revista Española de Salud Pública, 77(1)

Montoro, L.; Toledo, F.; Lijarcio, J.I. y Roca, J. (Coords.). (2006). Programa de Intervención, Sensibilización y Reeducación Vial - Manual del Formador. Madrid: Dirección General de Tráfico.

Organización Mundial de la Salud. (2004). Informe mundial sobre prevención de los traumatismos causados por el tránsito. Washington, D.C.: OPS.

Ozcoidi, M. (2001). Historia de los informes psicofísicos para conductores en Huesca. En: 100 años del Permiso de Conducción en España. Recopilación de trabajos Premiados (pp. 231-287) tomo I, Dirección General de Tráfico. Madrid.

Ozcoidi,M. (2004). Evolución Histórica de la Valoración de los Criterios de Aptitud. Universitat deValència: Tesis Doctoral.

Ozcoidi, M., Sanz, R., Reula, M.J. y Gonzalez Luque, J.C. (2002). La necesidad de mantener el permiso de conducir en los mayores como condicionante del estilo de vida en el medio rural. ANNALES 2002, U.N.E.D. (Universidad Nacional de Educación a Distancia), sede de Barbastro (Huesca). 293-393.

Prieto, JM, Fernández-Ballesteros, R., y Carpintero, H. (1994). Contemporary psychology in Spain. Annual Review of Psychology, 45, 51-78.

Saiz, M. y Saiz, D. (1996). Emilio Mira y la psicotecnia. En M. Saiz, D. Saiz (coords.) Personajes para una Historia de la Psicología en España. Barcelona: Ed. Autonoma/Piramide.

Saiz,D. y Saiz,M. (1998). La psicología aplicada en España. En F.Tortosa (Ed.), Historia de la Psicología Aplicada en Europa (pp 83-119). Revista de Historia de la Psicología, 19(1).
Santolaya, F., Berdullas, M. y Fernández Hermida (2002). Análisis del desarrollo de la Psicología Profesional en España. Papeles del Psicólogo. Época III, nº 82, 65-82.

Serra,J. (2003). El psicólogo español ante la prevención de los accidentes de tráfico. Un modelo de actuación para una historia. Universitat deValència: Tesis Doctoral.

Serra, J. y Tortosa, F. (2003). Una historia de la psicología de la seguridad vial en España. Valencia: Promolibro.

Soler, J. y Tortosa, F. (1987). Psicologia y Seguridad Vial en España: Una perspectiva histórica. En J. Soler y F. Tortosa Psicología y Tráfico (13-50). Valencia. Nau Llibres.

Soler i Dopff, C. (1930). La selecció dels conductors d'automobil per mitjans psicotécnics. Anals d'Orientació Professional, An IV (4), 319-321.

Terrasa,B. (2005). Psicología aeronáutica en España. Una historia y una panorámica actual. Universitat deValència: Tesis Doctoral.

Tortosa, F., Montoro, L. y Carbonell, E. (1989). Psicología y tráfico en España: 60 años de historia. Zaragoza. Librería General.

Tortosa, F. , Quintanilla, I. , Civera, C. y Diaz, R. (1993). Psychologie et profession en Espagne. Profils historiques et situation actuelle. Special issue: Applied Psycholoy in Europe: The professionalization process. European Review of Applied Psychology, 43(2), 123-139.

Tortosa, F., Esteban, C., Tejero,P. y Molero,C. (1995). La selección psicológica de los conductores en España: Desarrollo histórico y situación del modelo actual. En L. Montoro, E. Carbonell, J. Sanmartín, y F. Tortosa (Eds.). Seguridad Vial: Del factor humano a las nuevas tecnologías (pp. 375395). Madrid: Sintesis.

Tortosa, F., Civera, C. y Pastor, J. C. (2001). Una historia para un perfil profesional, el psicólogo experto en seguridad. Revista de Historia de la Psicología, 22(3-4), 543-559.

Tortosa, F. y Montoro, L. (2002). La psicología aplicada a la selección de conductores. Cien años salvando vidas. Psicothema, 14(4), 714-725.

Tortosa, F., Barjonet, P., Civera, C. y Montoro, L. (2003). Una historia de la psicologia del tráfico y el transporte en Europa. Anuario de Psicología, 34 (3), 401-41. 